\title{
GENERATION OF QUANTUM STATES OF LIGHT IN NONLINEAR ALGAAS CHIPS:
} ENGINEERING AND APPLICATIONS

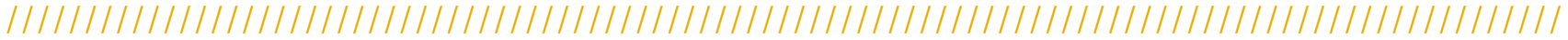

\section{Sara DUCCI ${ }^{1 *}$, Perola MILMAN ${ }^{1}$, Eleni DIAMANTI ${ }^{2}$}

${ }^{1}$ Laboratoire Matériaux et Phénomènes Quantiques, Université de Paris, CNRS-UMR 7162, Paris, France

${ }^{2}$ Sorbonne Université, CNRS, LIP6, 4 place Jussieu, F-75005 Paris, France

* sara.ducci@u-paris.fr

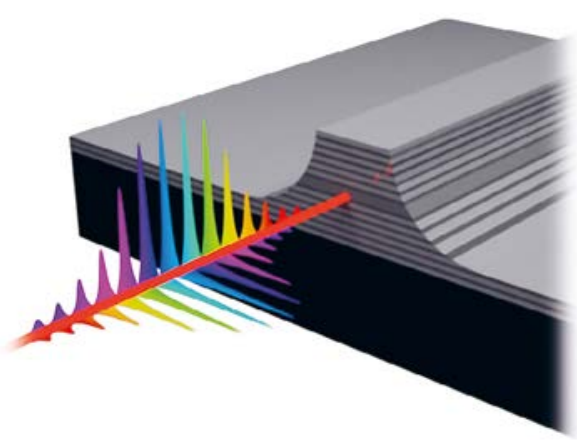

Photonic quantum technologies represent a promising platform for applications ranging from long-distance secure communications to the simulation of complex phenomena. Among the different material platforms, direct bandgap semiconductors offer a wide range of functionalities opening promising perspectives for the implementation of future quantum technologies. In this paper, we review our progress on the generation and manipulation of quantum states of light in nonlinear AlGaAs chips and their use in quantum networks.

https://doi.org/10.1051/photon/202110728

This is an Open Access article distributed under the terms of the Creative Commons Attribution License (http://creativecommons.org/licenses/by/4.0), which permits unrestricted use, distribution, and reproduction in any medium, provided the original work is properly cited.

\section{NONLINEAR QUANTUM PHOTONICS IN THE ALGAAS PLATFORM}

Integrated quantum photonics is playing a leading role in the development of quantum technologies and significant progress has been made using a wide variety of platforms for the generation, manipulation and detection of quantum states of light. Semiconductor materials, which are already at the basis of current classical communication and computation technologies, are ideal systems for the miniaturization and integration of several quantum components and important achievements have been reached by exploiting both the Si and the III-V platforms. In this context,
AlGaAs presents several assets [1,2]: high purity quantum states of light can be generated either using the single emitter approach (quantum dots) or by using nonlinear frequency conversion processes thanks to its strong nonlinearity ( $\chi^{2}$ of GaAs $~ 110 \mathrm{pm} / \mathrm{V}$ at $1550 \mathrm{~nm}$ ). In both cases, the direct band-gap of this material has allowed to demonstrate electrically injected sources, offering a clear advantage over optically driven ones in terms of portability, energy consumption, and integration. Photon manipulation and routing in quantum circuits thanks to electro-optical effects has also been demonstrated; finally, a full on-chip integration of generation, manipulation and detection of quantum states of light has been shown using this platform. In this paper, we focus on the generation of biphoton states exploiting the process of spontaneous parametric downconversion (SPDC) in AlGaAs waveguides; in this process, photons of an impinging pump laser beam of energy $\hbar \omega_{p}$ can be probabilistically converted into photon pairs, usually called signal and idler, of energy $\hbar \omega_{s}$ and $\hbar \omega_{i}$, respectively. In order to have a maximum efficiency in the frequency conversion, both energy and momentum have to be conserved. Despite its nondeterministic nature, this process is widely used to produce photon pairs and leads to devices working at room temperature and emitting photon pairs in the C-telecom band, thus 


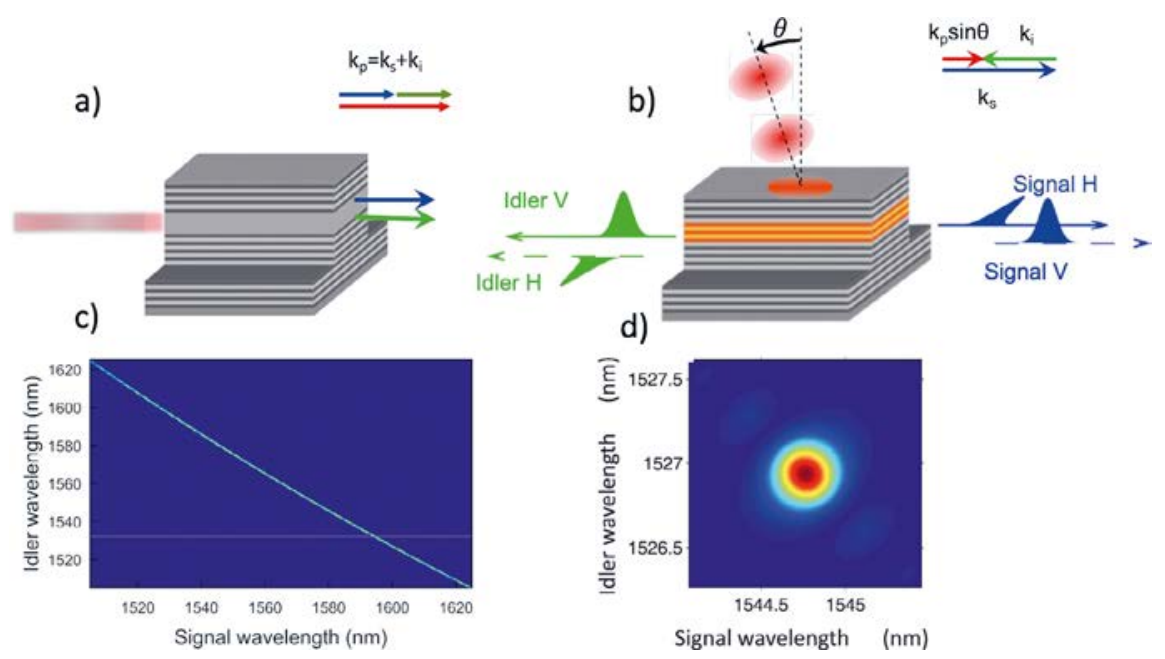

Figure 1. Top: Sketch of AlGaAs waveguides emitting photon pairs by SPDC based on two different phase-matching schemes. (a) Pump, signal and idler are collinear and co-propagating. (b) The pump beam is transverse with respect to the waveguide, and signal and idler are counterpropagating. Bottom: joint spectral intensity corresponding to co-propagating (c) and counterpropagating (d) phase-matching geometry.

facilitating their use in real world quantum information and communication protocols (see also the work done in the group of Prof. G. Weihs in Innsbruck, and Prof. A. Helmy in Toronto). In order to deal with the isotropic structure of this crystal, several solutions have been proposed to satisfy momentum conservation (called also phase-matching (PM) condition) in AlGaAs waveguides. Among these, the most advanced results in quantum states generation and manipulation have been obtained using two phase-matching geometries: a collinear modal PM scheme, in which the phase velocity mismatch is compensated by multimode waveguide dispersion, and a counterpropagating PM scheme, in which photon pairs exit from opposite waveguide's facets (Fig. 1). The choice of the PM scheme has a strong impact on the joint spectrum of the generated photon pairs: in the case of the collinear PM scheme, the photon pairs present a broadband and strongly

\section{Figure 2.}

Scanning electron microscopy image of an electrically injected device generating photon pairs by internal SPDC (a) and of a photonic chip integrating a heralded single photon source and a 50/50 coupler (b). In this device, a pump laser beam vertically incident on one of the input guides generates pairs of photons propagating in opposite directions. One photon of each pair is detected in arm $\mathrm{H}$ to herald the presence of its twin. The purity of the emitted photons is assessed by analyzing the temporal statistics of the photons transmitted by the waveguides at the output. Figure a) adapted from Technologies 4, 24 (2016) under CC license; Figure b) adapted from Photoniques 91, 25 (2018) under CC license.
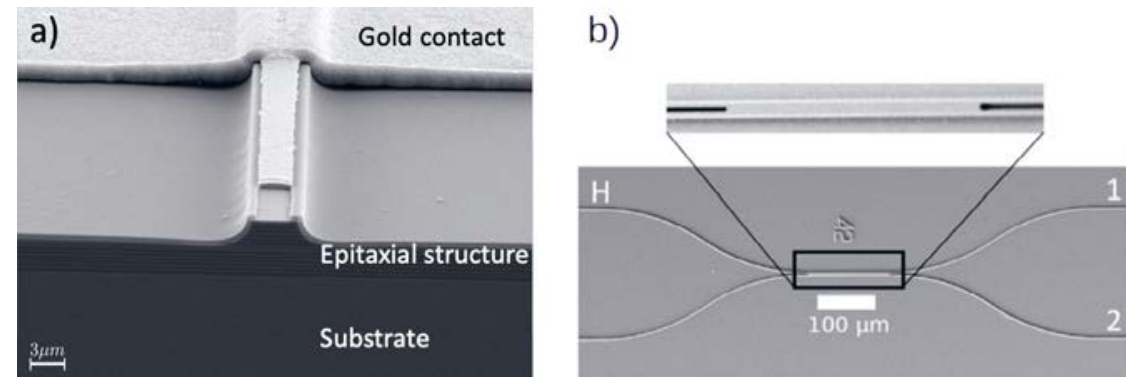


\section{COMPLETE SOLUTIONS FOR QUANTUM APPLICATIONS}

\section{Menlo Systems' optical frequency combs and ultra-stable lasers enable the second quantum revolution}

Quantum technology lets us exploit the laws of quantum mechanics for tasks like communication, computation, simulation, or sensing and metrology. As the second quantum revolution is ongoing, we expect to see the first novel quantum devices replace classical devices due to their superior performance.

There is a strong impetus to transform quantum technologies from fundamental research into a broadly accessible standard. Quantum communication promises a future with absolute security through quantum key distribution; quantum simulators and computers can perform calculations in seconds where the world's most powerful supercomputers would require decades; quantum technologies enable advanced medical imaging techniques. Further applications will likely arise that we cannot anticipate yet. The global market has realized the huge potential of quantum technologies. Menlo Systems, a pioneer in the field, provides commercial solutions for these novel challenges.

The link between photonics and quantum physics is obvious. Quantum simulation and computation use cold atoms and ions as qubits, labs worldwide use optical frequency combs and ultra-stable lasers in these types of experiments. Quantum communication often relies on single photons, which are generated with precisely synchronized femtosecond laser pulses in the near-infrared (-IR) spectral range. Quantum sensing and metrology require the highest stability and accuracy in frequency comb and laser technology. And - an application worth highlighting - optical atomic clocks are under way to replace the current definition of the second in the International System of Units (SI).
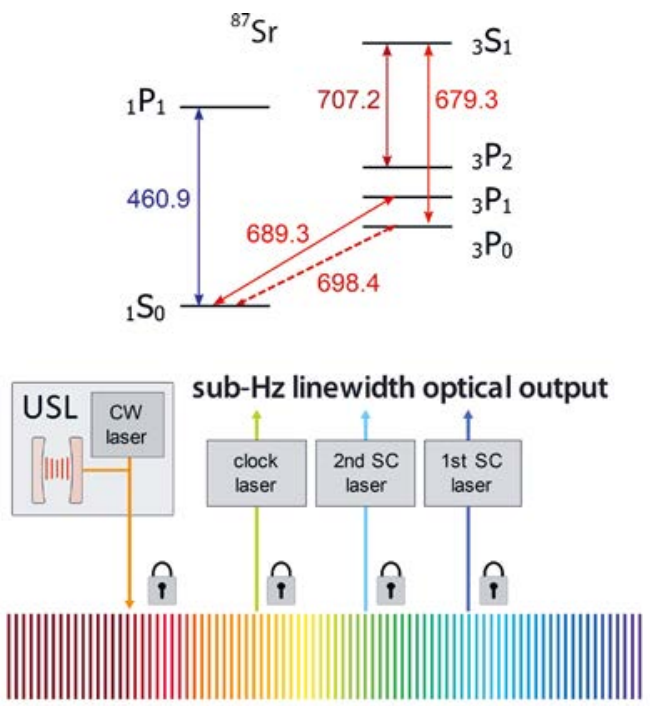

\begin{abstract}
Figure:
The hyperfine transitions in strontium (Sr) atoms with the ultra-narrow clock transition at 698.4 nm (upper part). A commercial FC1500Quantum system for optical clock applications contains an ultra-stable laser transferring its spectral purity onto an optical frequency comb and all other lasers which are also locked to the comb (lower part).
\end{abstract}

The transition frequency in optical clocks is on the order of hundreds of terahertz, corresponding to the visible or the ultraviolet region of the electromagnetic spectrum. Counting these optical frequencies is only possible using a frequency comb [1], a mode-locked laser with evenly spaced frequency modes within its optical spectrum. When referenced to a CW laser which is stabilized to a highfinesse optical cavity, the bandwidth of the comb lines narrows down to below 1 Hertz [2], corresponding to a stability of $10^{-15}$ or better. The newest generation of optical atomic clocks enabled by this technology reach an accuracy of $10^{-18}$ [3], two orders of magnitude higher than the best cesium atomic clock.
Menlo Systems' FC1500-Quantum is a complete CW laser system with an ultra-stable frequency comb. It provides several CW lasers for atom cooling, repumping, and addressing the sub-Hertz linewidth clock transition in atoms or ions used in optical clocks (see figure). The low phase noise obtained on the comb-disciplined CW lasers is essential for coherent gate manipulation in many atom optical quantum computing schemes and for fast and high-fidelity gate operations. The laser light is delivered via optical fiber to the "physics package" consisting of vacuum chamber with all the necessary optics and electronics components and the atoms. Labs no longer need to undergo the time consuming process of designing and building their own ultra-stable lasers. Eventually, the comb itself has two purposes: It acts as reference for all the lasers to maintain their narrow linewidth, and it is the clockwork that transfers the optical frequency's spectral purity to the microwave region, or to a different optical frequency [4].

\section{CONTACT:}

Menlo Systems $\mathrm{GmbH}$

Bunsenstraße 5

82152 Martinsried, Germany

Phone: +49 891891660

Fax: +49 89189166111

sales@menlosystems.com

www.menlosystems.com 
anti-correlated joint spectrum; in the case of counterpropagating PM scheme the photon pairs are characterized by a narrowband spectrum and their spectral correlations can be engineered through the pump beam profile. One of the important advantages of the collinear PM scheme is its compatibility with electrical injection allowing for the monolithic integration of the pump laser and the nonlinear medium. In this device, working at room temperature, the active medium is a quantum well inserted in the core of the structure; the electrical injection of the device generates the laser emission which is converted in photon pairs in the C-telecom band via intracavity SPDC (Fig. 2a). A further step towards a fully quantum integrated photonic circuit has been done by demonstrating the monolithic integration of a heralded single photon source and beamsplitters using the counterpropagating PM scheme; this device has been used to implement an integrated Hanbury-Brown and Twiss experiment confirming the single photon operation (Fig. 2b). The quality of the quantum state generated in both PM schemes has been analyzed through measurements of indistinguishability (using for example Hong-Ou-Mandel interferometers), quantum correlations and entanglement - between the generated photons - in several degrees of freedom like polarization, energy-time and time bins. In this regard we note that the group velocity mismatch between orthogonally polarized photons in the AlGaAs chip is so small that no off-chip walk-off compensation is required to obtain polarization entanglement. This is a key feature enabling the direct use of the emitted pairs at the output of the chip, opening the way to its easy integration into simple and robust architectures.

\section{QUANTUM STATE ENGINEERING}

In recent years, entanglement in high-dimensional degrees of freedom of photons has attracted a growing attention as a means to increase the throughput and security of quantum communication and enhance flexibility in quantum computing. In this context, frequency is a particularly attractive degree of freedom thanks to its robustness to propagation in optical fibers and its capability to convey a large amount of quantum information into a single spatial mode. This provides a strong motivation for the development of efficient and scalable methods to generate and manipulate frequency-encoded quantum states. In this respect, the counterpropagating PM scheme allows for an extremely high versatility in quantum state engineering. Indeed, tailoring the spatial profile (intensity and phase) of the pump beam enables the control of the spectral correlations and wavefunction symmetry of the photon pairs directly at the generation stage, without any post-selection. In particular, tuning the pump beam waist allows to produce correlated, anti-correlated and separable frequency states, while modifying the spatial phase profile allows to switch between symmetric and antisymmetric spectral wavefunctions and modify the exchange statistics of the photons (see Fig. 3) [3]. These results could be harnessed to study the effect of exchange statistics in various quantum simulation

\section{CVQKD USING IXBLUE MODULATORS AND MATCHING RF AMPLIFIERS}

Quantum Key Distribution (QKD) offers a forever privacy guaranteed by the laws of physics. A spy trying to intercept some information is detected before a message is even sent. And this is achieved simply by adapting the emitter and receiver hardware of an optical link (no need to send guards all along your optical fiber).

In practice, QKD is achieved with optical telecommunication links, either via optical fibers or the propagation of light in vacuum (or the atmosphere) for satellite links where iXblue modulators are used.

An example of Continuous Variable QKD (CVQKD) is given. The information is encoded in both the amplitude and the phase of laser pulses using iXblue solutions: two amplitude modulation blocks AM1 and AM2 are cascaded with a phase modulation PM1.

- Using an AWG, a first modulation block AM1 is used to generate short optical pulses. Using iXblue NIR-MX800, MX1300 and MXER high contrast and wide bandwidth amplitude modulators, very short optical pulses width from $70 \mathrm{ps}$ can be achieved at $850 \mathrm{~nm}, 1310 \mathrm{~nm}$ and $1550 \mathrm{~nm}$ respectively. The modulator is combined with the driver DR-VE-10-MO which can be set either as a limiting or linear amplifier for either square or gaussian pulse waveforms. Using iXblue bias controller MBC-PL-LAB, a high pulse contrast stability is obtained for frequency repetition rates up to several GHz.

- An additional modulation block AM2 generates the random amplitude required for each pulse in CVQKD. This is achieved using the MXAN-LN and the highly linear DR-VE-10-MO.

- A phase modulator PM1 sets the phase of each pulse. The MPZ-LN-01 (coming with more than $3 \mathrm{GHz}$ electro-optical bandwidth) or the MPZ-LN-10 (typical $16 \mathrm{GHz}$ of bandwidth) is used in combination with the driver DR-AN-10-HO to continuously modulate the phase over the range 0 to $2 \pi$.

iXblue provides modulation solutions to QKD manufacturers and to research institutions. In addition to the solutions listed above, iXblue also offers polarization switches and pulse pickers. iXblue is also participating to the OpenQKD consortium. By offering dedicated modulators, bias controllers and RF drivers, pulse-pickers for receiver temporal pulse selection, iXblue is proud to contribute efficiently to the deployment QKD.

\section{More info on iXblue Photonics website}

Contacts: Baptiste Gouraud - Hervé Gouraud contact.photonics@ixblue.com 
a)

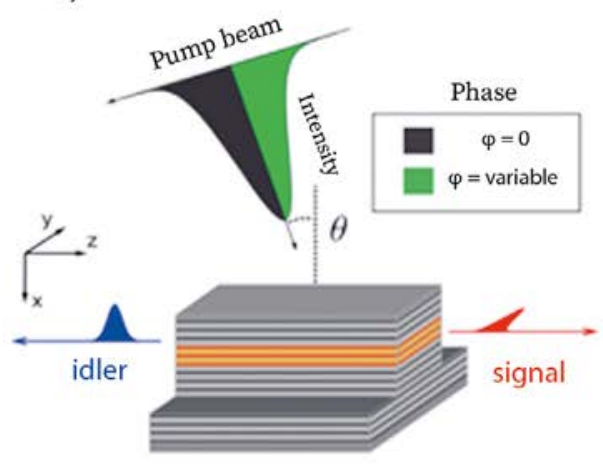

b)

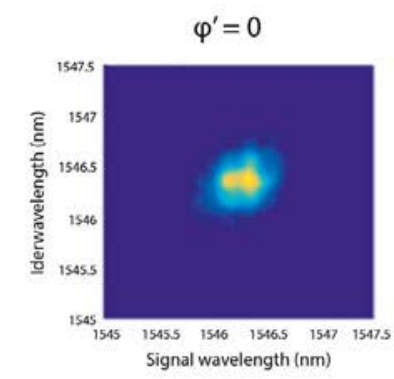

d)

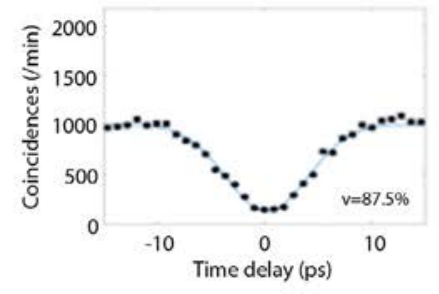

c)

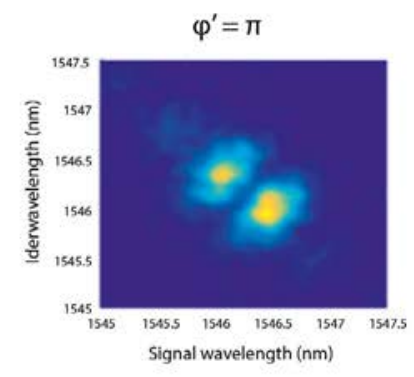

e)

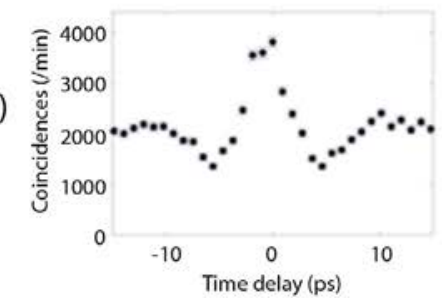

problems, and to implement communication and computation protocols exploiting antisymmetric high-dimensional quantum states. Another interesting aspect related to $\mathrm{AlGaAs}$ chips is the inherent cavity effect arising from the refractive index contrast between semiconductor and air at the waveguide's facets; in devices based on the collinear PM scheme, this leads to the generation of biphoton frequency combs spanning several tens of nanometers (Fig. 4). These states, that have also been generated in integrated nonlinear optical resonators based on third order nonlinear optical processes, consist of discrete spectral modes and are characterized by a biphoton phase coherence guaranteed by the generation process. They constitute entangled

\section{Figure 3:}

a) Sketch illustrating the tailoring of the pump profile to engineer the signal/idler exchange statistics in the device based on the transverse pump configuration; b, c) measured joint spectrum for different values of the phase shift imposed to the pump beam; d, e) corresponding two-photon interference measured in a Hong-Ou-Mandel interferometer.

qudits, i.e. d-level quantum systems, providing promising applications for quantum information processing [4]. In this context, we have shown that the interplay between cavity effects and a temporal delay between photons of a pair allows to switch from symmetric to anti-symmetric high-dimensional states opening the way to the
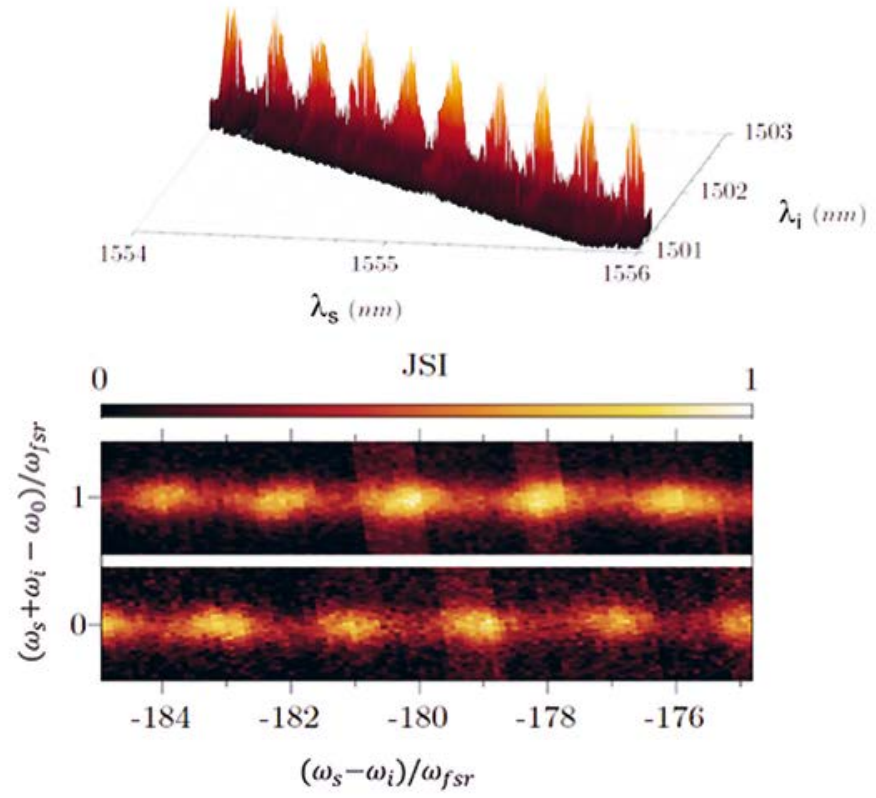

implementation of qudit teleportation, logic gates, as well as dense coding and state discrimination [5].

\section{APPLICATIONS IN QUANTUM INFORMATION AND \\ COMMUNICATION PROTOCOLS}

The richness and the quality of the quantum states generated and controlled in AlGaAs chips in terms of degrees of freedom, dimensionality, possibility to have simultaneous entanglement in different degrees of freedom (namely hyperentanglement) opens many perspectives for both fundamental research and for the implementation of future quantum technologies.

For example, continuous degrees of freedom of photons display a perfect analogy with the continuous variables (CV) of a multiphoton single mode of the electromagnetic field, which makes them a promising platform to realize $\mathrm{CV}$

\section{Figure 4:}

Top: Experimental measurement of the Joint Spectral Intensity of the state generated by a device based on a collinear phase-matching geometry, showing a frequency comb structure. Bottom: generation of resonant and antiresonant biphoton frequency combs by tuning the pump beam frequency. This control, combined to the insertion of a temporal delay between photons of a pair, allows to switch from symmetric to anti-symmetric quantum frequency states. Figure adapted from [5] under Creative Common License. 
WORLD

CLASS $\times 1 \%$

LASERS

\& FIBERS $\%$

$\therefore$ a

Ultrafast lasers Photonic crystal fibers Single-frequency lasers Supercontinuum white light tasers
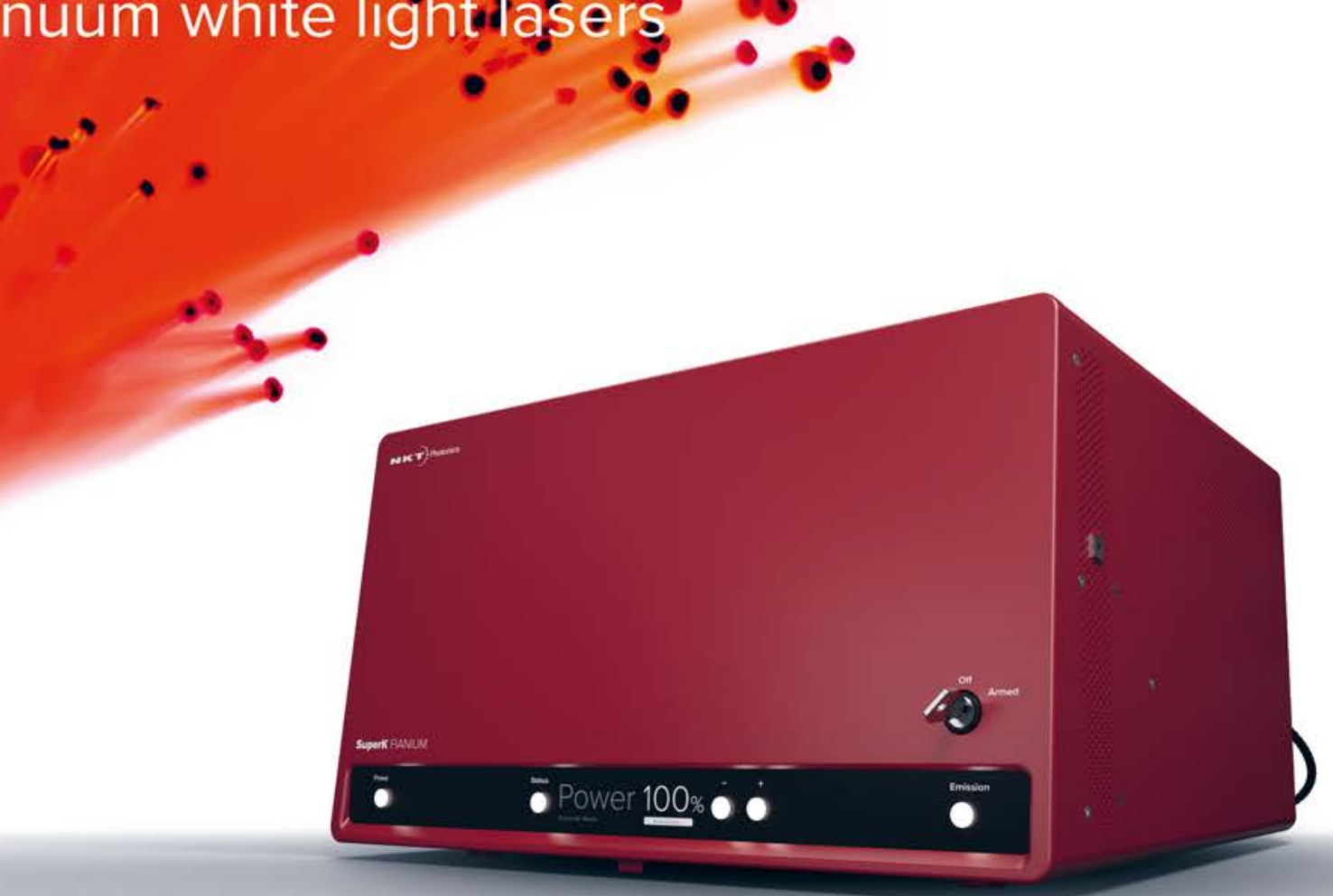


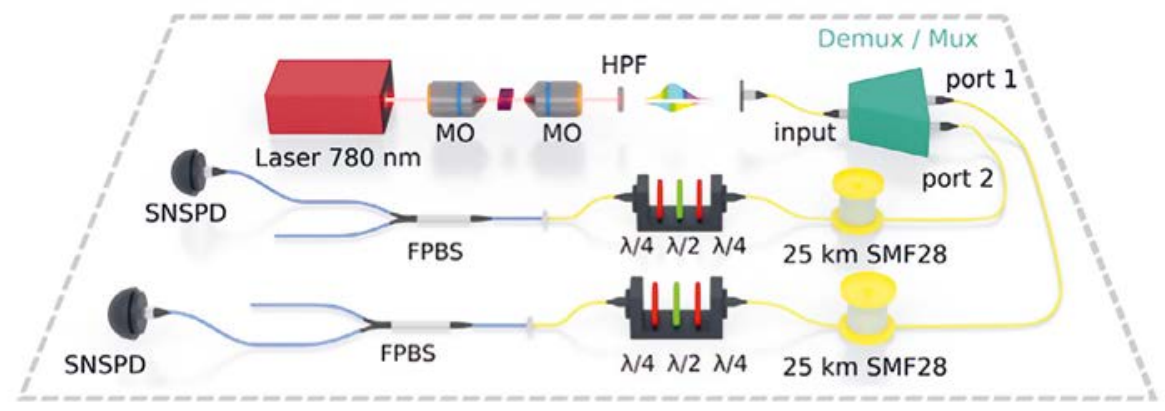

quantum information protocols in the few-photon regime. In this regard, we have shown that biphoton frequency combs can be used to generate, manipulate, and detect grid states encoded in time ( $\mathrm{t}$ ) and frequency $(\omega)$. The redundancy of information arising from the periodicity of time-frequency space gives these states a particular robustness against time and frequency shifts, opening the way to the implementation of quantum error correction operations [6]. Also, continuous degrees of freedom of single photons are relatively easy to engineer using spatial light modulators acting on the pump beam as well as measurements and time delays, opening the perspective of extending the range of application of frequency and time degrees of freedom to different quantum information related protocols for continuous variables, as for instance quantum communications and quantum metrology.

In the context of quantum communications, the ability to maintain high quality polarization entanglement over a broad spectral region in the C-telecom band makes AlGaAs sources particularly appealing. Recently, the combination of these devices with industry-grade wavelength division multiplexing techniques has allowed us to build a reconfigurable, fully connected entanglement distribution quantum network between up to 8 users in a resource-optimized quantum network topology (Fig. 5). We have demonstrated that the lower bound on the fidelity of the entangled state generated by our chip with respect to an

\begin{abstract}
Figure 5:
Sketch of the quantum network architecture exploiting broadband polarization entangled states produced by an AlGaAs chip. The setup consists of three stages: entanglement generation, frequency demultiplexing/ multiplexing of the generated signal, and a distribution stage including fiber links, a polarization analysis stage fibered polarizing beam splitters (FPBS) and superconducting nanowire single photon detectors (SNSPD).
\end{abstract}

ideal Bell state stays above $95 \%$ over a $26 \mathrm{~nm}$ wide spectral range around biphoton degeneracy and above $85 \%$ over a $60 \mathrm{~nm}$ range. The performance of the network has been benchmarked by running an entanglement-based quantum key distribution protocol between two users across fibered optical links of up to $50 \mathrm{~km}$ and we extrapolate a positive key rate for distances of up to $75 \mathrm{~km}$ in both symmetric and asymmetric configurations opening the way to the implementation of our architecture in metropolitan-scale quantum communication networks [7].

\section{CONCLUSION}

Nonlinear AlGaAs devices allow to generate pure quantum states of light in two and higher dimensions with a high level of flexibility and on-chip control; they work at room temperature, telecom wavelength and are compatible with electrical injection. These results open a wide variety of perspectives for the implementation of future quantum technologies. The level of integration can be pushed further by implementing additional on-chip functionalities such as polarizing beam-splitters or photon routers exploiting the electro-optics effect. Hybrid devices integrating a III-V generation stage with Si-based photonics circuits are also promising candidates for fully integrated quantum integrated photonic chips bringing together the best of the two worlds. We also note that recently, entangled-photon -pair generation has been demonstrated using third order nonlinear effects in AlGaAs-oninsulator devices, opening another promising approach for all-on-chip quantum information applications. From the point of view of fundamental research, the obtained level of control and engineering on the biphoton time-frequency phase space opens the way to the generation and manipulation of exotic states, like Schrödinger cat-like state, compass states or grid states which can find interesting applications in metrology or quantum error correction codes. Finally, the recent demonstration of flexible entanglement-based secure communication using AlGaAs chips offers a promising route to the deployment of scalable quantum network architectures which could benefit from other features of the generated quantum states, such as their biphoton comb structure as well as the combined exploitation of both polarization and frequency entanglement.

REFERENCES

[1] C. P. Dietrich et al., Laser Photonics Rev. 10,870 (2016)

[2] A. Orieux et al., Rep. Prog. Phys. 80076001 (2017)

[3] S. Francesconi et al., Optica 7, 313 (2020)

[4] M. Kues et al., Nat. Photonics 13, 170 (2019)

[5] G. Maltese et al., Npj Quantum Inf. 6, $13(2020)$

[6] N. Fabre et al., Phys. Rev. A 102,

012607 (2020)

[7] F. Appas et al., arXiv:2102.04835v1 (2021) 